\title{
Früher war alles besser?
}

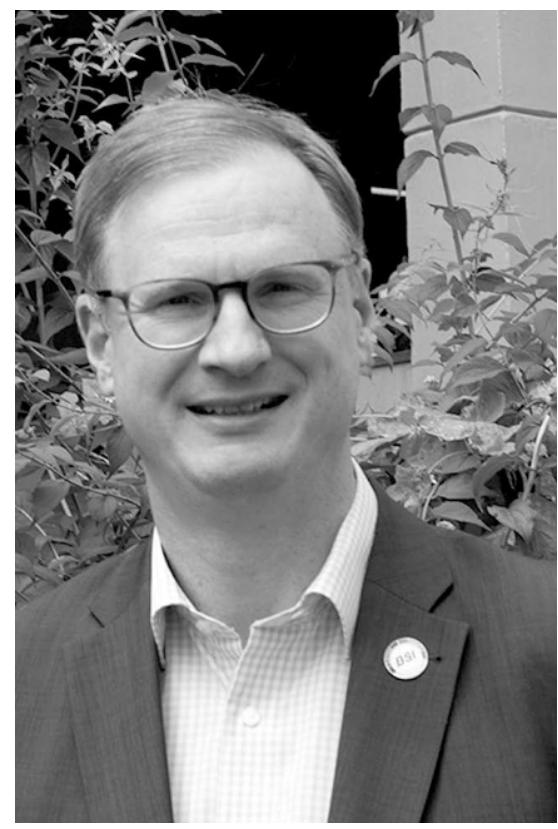

Gilt dies für die IT-Sicherheit? Sicherlich erscheint es richtig, dass es früher nicht besser aber durchaus anders war. Anders als heute und auch kontinuierlich anders in der Zukunft. Jede Zeit braucht ihre Antworten, wie auch die Politik erkannt hat. Das ist ebenso gültig für eine Informationstechnik, die sich von Jahr zu Jahr schneller entwickelt und damit zu einer disruptiven Triebfeder der Digitalisierung in einer globalen Ausprägung geworden ist. Eine Informationstechnik, die im Unterschied zu früher um Potenzen stärker global als national (aus Deutschland) beeinflusst ist.

IT-Sicherheit und Informationssicherheit als wichtige Eigenschaften der modernen Informationstechnik bleiben zwar über die Zeiten hinweg ihren Zielen treu, aber mit welchen Instrumenten, Werkzeugen, Methoden und Prozessen sie erreicht werden, ist ebenfalls einem Wandel unterworfen. Was für die Informationstechnik und ihre Sicherheit allgemein gilt, hat gleich große Bedeutung für die Nutzung im Bereich der Verwaltung, wenn es darum geht, für den Bereich der informationssichernden IT-Systeme für nationale Verschlusssachen ("VS" - oder auch klassifizierten Informationen im nationalen Geheimschutz) Lösungen bereitzustellen. Dieses Arbeitsgebiet, welches häufig unter dem Stichwort "Hochsicherheit" rangiert, muss sich ebenfalls auf die umwälzenden Veränderungen im Bereich der Informationstechnologie einstellen. Konnten in der Vergangenheit die staatlichen IT-Infrastrukturen, die für die Verarbeitung und Übertragung von klassifizierten Informationen genutzt wurden, häufig noch als separierter Bereich implementiert werden, so ist im Zeitalter der durchdringenden Informationsverarbeitung (z.B.Cloud Computing) dieses praktisch kaum mehr durchsetzbar. Nicht nur, weil die behördlichen Nutzer eben auch auf einen sehr umfassenden und kaum separierbaren Informationsraum zugreifen müssen, um ihren jeweiligen Auftrag umsetzen zu können, sondern auch deswegen, weil der in der Vergangenheit vorhandene "Sondermarkt" für vertrauenswürdige nationale IT-Produkte für die bis dahin vorhandenen wohlstrukturierte IT-Infrastrukturen mit Perimeterschutz und klarer Trennung zwischen Innen und Außen kontinuierlich schrumpft.

Zweifellos ist eine der drängendsten Fragen der Bedarfsträger im Bereich des Geheimschutzes, wie man Innovationen, die sich in der kommerziell verfügbaren Informationstechnik zeigen, möglichst schnell auch im Bereich der VS-IT zur Nutzung bereitstellen kann. Wichtig ist, die richtigen Randbedingungen, Erfolgskriterien und Aktivitäten mit den in diesem Feld tätigen Akteuren zu bestimmen. Neben einer smarten Regulierung des Geheimschutzes und der verlässlichen Nachfrage des staatlichen Bereichs nach vertrauenswürdigen IT-Systemen ist unter anderem auch die unternehmerische Innovationskraft der deutschen IT-Sicherheitsindustrie gefragt. Ich bin mir sicher, je besser sich die Beiträge der Akteure zusammenfügen, desto leistungsfähigere Lösungen werden wir finden und damit auch wirksam zur nationalen digitalen Souveränität in der IT-Sicherheit beitragen.

Das vorliegende Heft ist darauf ausgerichtet, Antwortbeiträge und Lösungsvorschläge zu einigen aufgeworfenen Fragen zu formulieren. Es spannt dazu einen Bogen von den rechtlichen Grundlagen des Geheimschutzes, der Zulassung und Evaluierung von Produkten bis hin zur Bereitstellung von zukünftigen IT-Sicherheitsprodukten in einem innovativen Umfeld und Berücksichtigung des IT-Sicherheitsmarktes. Dabei werden auch die für den staatlichen Geheimschutz wichtigen technologischen Themen, wie Kryptografie, Künstliche Intelligenz und Cloud Computing diskutiert.

\section{Günter Welsch}

